Pacific Journal of Mathematics

ON THE RADIAL LIMITS OF BLASCHKE PRODUCTS 


\title{
ON THE RADIAL LIMITS OF BLASCHKE PRODUCTS
}

\author{
G. R. Maclane and F. B. RYan
}

1. Introduction. As is well known, a Blaschke product $f(z)$ in $\{|z|<1\}$ has radial limits $f\left(e^{i \theta}\right)$ of modulus one almost everywhere on $\{|z|=1\}$. The object of the present paper is to give a partial answer to the question: how many times does $f(z)$ assume a given radial limit? We shall prove the following theorem.

Theorem A. Let $E$ be a given closed set on $\{|w|=1\}$ and let $E^{\prime}$ be the complement of $E$ relative to $\{|w|=1\}$. Then there exists a Blaschke product $f(z)$, all of whose radial limits are of modulus one, and such that the set

$$
L(\beta)=\left\{\theta \mid f\left(e^{i \theta}\right)=e^{i \beta}\right\}
$$

has the power of the continuum for $e^{i \beta} \in E$ and is countable for $e^{i \beta} \in E^{\prime}$.

Theorem $\mathrm{A}$ is a condensed statement of what we shall actually prove; Theorems 1, 2, and 3 contain somewhat more information on $f(z)$. The method of proof is to construct a suitable regularly-branched covering $\mathscr{W}$ of $\{|w|<1\}$, corresponding to an automorphic function $w=f(z)$, and then use the geometry of $\mathscr{W}$ to obtain our results.

The question naturally arises as to whether one could prove Theorem A directly. That is: could one produce an $f(z)$ with the desired properties by exhibiting its zeros instead of defining $f(z)$ by means of a surface $\mathscr{W}$ ? The answer to this question does not seem to be obvious.

2. The surface $\mathscr{W}$. Let $E$ be a given nonvoid closed subset of $\{|w|=1\}$ and let $\left\{a_{n}\right\}_{1}^{\circ}$ be an infinite sequence of points in $\{|w|<1\}$ whose derived set is $E$. Clearly, we may assume that $a_{n} \neq 0$ and

$$
\arg a_{m} \neq \arg a_{n} \quad(m \neq n) .
$$

Let $\mathscr{W}$ be the simply-connected unbordered covering of $\{|w|<1\}$ which is regularly-branched over the points $\left\{a_{n}\right\}$ with all branch points of multiplicity 2 . It is well known $[2,3,6]$ that such a covering, with any specified multiplicity or signature for each $a_{n}$, exists and is unique. Instead of appealing to the general theory of regularly-branched coverings, we shall construct the surface $\mathscr{W}$ directly, since the details of the construction play a role in the proof of Theorem A.

Received September 29, 1961. The research of the first author was supported by the United States Air Force through the Air Force Office of Scientific Research of the Air Research and Development Command, under Contract No. AF 49 (638)-205. 
Let $C_{n}$ be the radial segment $\arg w=\arg a_{n},\left|a_{n}\right| \leqq|w|<1$. The $C_{n}$ are disjoint because of (1). We make cuts in $\{|w|<1\}$ along each $C_{n}$ and so obtain a alit disc $W$, copies of which are joined together, according to the following specifications, to form the surface.

$0^{\text {th }}$ level. The surface $\mathscr{W}_{0}$ consists of just one slit disc $W$. Note that $\mathscr{W}_{0}$ is simply-connected.

$1^{\text {st }}$ level. The surface $\mathscr{W}_{1}$ is obtained by adjoining an infinite sequence of distinct copies of $W$, namely $W(1), W(2), \cdots$, to $\mathscr{W}_{0} . \quad W\left(n_{1}\right)$ is joined to $\mathscr{W}_{0}$ along $C_{n_{1}}$ so as to form a first-order branch-point over $a_{n_{1}}$. The surface $\mathscr{W}_{1}=\mathscr{W}_{0} \cup \bigcup_{n} W(n)$ is simply-connected; for by adjoining the $W(n)$ one at a time we obtain an increasing sequence of simply-connected surfaces which exhaust $\mathscr{W}_{1}$. We denote by $\chi\left(n_{1}\right)$ the curve in $\mathscr{W}_{1}$ along which $W\left(n_{1}\right)$ and $\mathscr{W}_{0}$ are identified.

$2^{\text {nd }}$ level. Along each free slit on the boundary of $\mathscr{W}_{1}$ we adjoin a copy of $W$. More precisely, the sheet $W\left(n_{1}, n_{2}\right)$ is adjoined to $W\left(n_{1}\right)$ along the cut $C_{n_{2}}$ in $W\left(n_{1}\right)$. The added sheets correspond one-to-one with all pairs $\left(n_{1}, n_{2}\right)$ of positive integers such that $n_{1} \neq n_{2}$. Again we see that the surface $\mathscr{W}_{2}=\mathscr{W}_{1} \cup \cup W\left(n_{1}, n_{2}\right)$ is simply-connected. The curve over $C_{n_{2}}$ along which $W\left(n_{1}\right)$ and $W\left(n_{1}, n_{2}\right)$ are joined is denoted by $\chi\left(n_{1} n_{2}\right)$.

$k^{\text {th }}$ level. Continuing the construction, the surface $\mathscr{W}_{k}$ consists of $\mathscr{W}_{k-1}$ and copies of $W$ denoted by $W\left(n_{1}, n_{2}, \cdots, n_{k}\right), \quad n_{i} \neq n_{i+1}$, which are joined to $\mathscr{W}_{k-1} ; W\left(n_{1}, \cdots, n_{k}\right)$ is adjoined to $W\left(n_{1}, \cdots, n_{k-1}\right)$ along the cut $C_{n_{k}}$ in $W\left(n_{1}, \cdots, n_{k-1}\right)$. Denote the curve along which those two sheets are joined by $\chi\left(n_{1}, n_{2}, \cdots, n_{k}\right)$. Clearly $\mathscr{W}_{k}$ is simply-connected.

We take the surface $\mathscr{W}$ to be $\lim \mathscr{W}_{k}$ as $k \rightarrow \infty$; it is clear that $\mathscr{W}$ is simply-connected as $\mathscr{W}_{k} \uparrow \mathscr{W}$. With the natural projection map onto $\{|w|<1\}$ it is clear that $\mathscr{W}$ is a regularly-branched, unbordered, covering of $\{|w|<1\}$. All points of $\mathscr{W}$ over the $a_{n}$ are branch-points of multiplicity 2 , and $\mathscr{W}$ has no other branch-points.

3. The function $f(z)$. Since $\mathscr{W}$ is a covering of $\{|w|<1\}$ it is hyperbolic. Let $w=f(z)$ be the holomorphic function which maps $\{|z|<1\}$ onto $\mathscr{W}$, with $f(0)=0 \in \mathscr{W}_{0}$ and $f^{\prime}(0)>0$. Clearly $|f(z)|<1$. The radial limits of $f(z)$ are all of modulus one, since if this were not the case a boundary point of $\{|z|<1\}$ would correspond to an interior point of $\mathscr{W}$ which is unbordered. Thus $f(z)$ is of class $U$ [5, p. 32]. Applying Frostman's theorem [5, p. 33] we see that $f(z)$ is a Blaschke product.

Also, $f(z)$ is an automorphic function with respect to a Fuschian group $F$, since the decktransformations of $\mathscr{W}$ correspond to linear 
transformations preserving $\{|z|<1\}$. It is easily shown that if $E=$ $\{|w|=1\}$ then $F$ is of the first kind: the limit points of $F$ fill $\{|z|=1\}$. If $E \neq\{|w|=1\}$ then $F$ is of the second kind: the set of limit points of $F$ is a perfect nowhere dense subset of $\{|z|=1\}$.

The sheets $W\left(n_{1}, n_{2}, \cdots, n_{k}\right)$ of $\mathscr{W}$ correspond to a set of fundamental regions $R\left(n_{1}, \cdots, n_{k}\right)$ of $F$. These are the fundamental regions which play a role in the proof; since these are defined via the function $f$ it is not clear that they are the same as the fundamental regions obtained by any of the usual constructions in terms of $F$. Hence we must derive some properties of these regions.

4. Properties of the fundamental regions. For convenience we reduce the notations $W\left(n_{1}, \cdots, n_{k}\right), R\left(n_{1}, \cdots, n_{k}\right)$, and $\chi\left(n_{1}, \cdots, n_{k}\right)$ to $W, R$, and $\chi$ respectively. To each curve $\chi$ in $\mathscr{W}$ there corresponds a simple arc $X$ in $\{|z|<1\}$. It is evident that the fundamental regions $R$ are bounded by the $X$ 's and points of $\{|z|=1\}$. We proceed with an investigation of the $X$ 's.

First, each $X$ ends at two distinct points of $\{|z|=1\}$. The two linear pieces of $\chi$ correspond to two simple ares $X^{\prime}$ and $X^{\prime \prime}$, and $f(z)$ tends to a limit as $|z| \rightarrow 1$ on $X^{\prime}$ and $X^{\prime \prime}$. Then by Koebe's lemma $\left[1\right.$, p. 213] each of $X^{\prime}$ and $X^{\prime \prime}$ must tend to a definite point of $\{|z|=1\}$. The end points of $X^{\prime}$ and $X^{\prime \prime}$ must be distinct. If not, let $D$ be that part of $\{|z|<1\}$ bounded by $X$ and a single point $b$ on $\{|z|=1\}$. Then the part of $\mathscr{W}$ corresponding to $D$ will contain an infinite number of sheets $W$ joined along various $\chi$ 's, which correspond to $X$ 's, all ending at $b$. Thus $f(z)$ would have infinitely many distinct asymptotic values, namely $\exp \left(i \arg a_{n}\right)$, at $b$; but this would contradict the theorem of Lindelof $[4$, p. 9] to the effect that a bounded holomorphic function can have at most one asymptotic value at a given point.

Thus each $X$ is a crosscut of $\{|z|<1\}$. A second property is that no two $X$ 's have a common endpoint. To see this, suppose $X_{1}$ and $X_{2}$ are two distinct $X$ 's with a common endpoint $b$ on $\{|z|=1\}$. Let the corresponding curves $\chi_{1}$ and $\chi_{2}$ in $\mathscr{W}$ end at points $\alpha_{1}$ and $\alpha_{2}$, respectively, over $\{|w|=1\}$. If $\alpha_{1} \neq \alpha_{2}$ then we would again have a contradiction of Lindelöf's theorem. Now suppose $\alpha_{1}=\alpha_{2}$. We may construct a sequence of $\operatorname{arcs} \Delta_{n}$ in $\{|z|<1\}$, each joining a point of $X_{1}$ to a point of $X_{2}$, such that diam $\Delta_{n} \rightarrow 0$. Since by Lindelöf's theorem $f(z) \rightarrow \alpha_{1}$ uniformly between $X_{1}$ and $X_{2}$ we may also require diam $\left\{f\left(\Delta_{n}\right)\right\}<1 / n$. But from the structure of $\mathscr{W}$ it is clear that there exists a curve $\chi$ on $\mathscr{W}$., with endpoint $\neq \alpha_{1}$, such that any curve on $\mathscr{W}$, joining a point of $\chi_{1}$ to a point of $\chi_{2}$, must intersect $\chi$. Since the projection of $\chi$ into $\{|w|<1\}$ and the common projection of $\chi_{1}$ and $\chi_{1}$ are a positive distance $\delta$ apart, we must have diam $\left\{f\left(\Delta_{n}\right)\right\} \geqq \delta$, which is incompatible with diam $\left\{f\left(\Delta_{n}\right)\right\}<1 / n$. 
Next, for any $\varepsilon>0$, the set $S=\{X \mid \operatorname{diam} X>\varepsilon\}$ is finite. For, any disc $\{|z|<1-\delta\}$ intersects only a finite number of the $X$ 's. Hence if $S$ were infinite there would exist an infinite sequence $\left\{X_{n}\right\}_{1}^{\infty}$ of distinct crosscuts and a nondegenerate arc $\Lambda$ on $\{z \mid=1\}$ such that the radius joining $z=0$ to an arbitrary point of $\Lambda$ crosses every $X_{n}$. Now any radial limit $f\left(e^{i \theta}\right)=e^{i \alpha}, e^{i \theta} \in \Lambda$, forces the $\chi_{n}$, corresponding to $X_{n}$ and ending at $e^{i \alpha_{n}}$, to satisfy $\alpha_{n} \rightarrow \alpha$. But then $f\left(e^{i \theta}\right)=e^{i \alpha}$ for almost all $e^{i \theta} \in \Lambda$, which contradicts the theorem of F. and M. Riesz. The point of this paragraph is that if $b$ is a limit point of $F$, then any neighborhood of $b$ contains infinitely many complete fundamental regions $R$. There are at least some examples of Fuchsian groups possessing a set of fundamental regions (connected) whose diameters are bounded away from zero.

\section{Properties of $f(z)$ on the boundary.}

THEOREM 1. Let $b$ be a limit point of $F, U$ a neighborhood of $b$, and let $e^{i \alpha} \in E$. Then the set

$$
U \cap L(\alpha)
$$

has the power of the continuum.

Proof. There exists a cross-cut $X$, corresponding to the curve $\chi$ in $\mathscr{W}$, which separates $\{z \mid<1\}$ into two domains, one of which, $D$, is contained in $U$. The corresponding part, $\mathscr{D}$, of $\mathscr{W}$ contains infinitely many sheets. In $\{|w|<1\}$ we may select among the $\operatorname{arcs} C_{n}$ two sequences, $\left\{C_{n}(0)\right\}_{1}^{\infty}$, and $\left\{C_{n}(1)\right\}_{1}^{\infty}$, which satisfy either the following three conditions

(2) the lengths of the $C_{n}(0)$ and $C_{n}(1)$ tend to zero,

(3) $\arg C_{n}(0) \downarrow \alpha$, and arg $C_{n}(1) \downarrow \alpha$,

(4) $\arg C_{n+1}(0)<\arg C_{n}(1)<\arg C_{n}(0)$;

or the same conditions with the arrows in (3) and the inequalities in (4) reversed. Such sequences $\left\{C_{n}(\varepsilon)\right\}, \varepsilon=0,1$ exist because of $e^{i \alpha} \in E$, the initial choice of $\left\{a_{n}\right\}$, and (1).

Now let $\Gamma(\varepsilon)=\Gamma\left(\varepsilon_{1}, \varepsilon_{2}, \cdots\right), \varepsilon_{i}=0,1$, be an arc in $\mathscr{D}$ with the properties:

(5) $\Gamma(\varepsilon)$ crosses, in order, curves $\chi$ in $\mathscr{D}$ over the arcs $C_{1}\left(\varepsilon_{1}\right), C_{2}\left(\varepsilon_{2}\right)$, $C_{3}\left(\varepsilon_{3}\right), \cdots$, and meets no other $\chi$ 's.

(6) $\Gamma(\varepsilon)$ tends to a point on the boundary of $\mathscr{D}$ over $e^{i \alpha}$.

This construction of $\Gamma(\varepsilon)$ is possible by (2), (3), (4), and since all the curves $\chi$ over $\alpha<\arg w<\alpha+\delta, \delta=\delta(\eta)$, are of length $<\eta . \quad \Gamma(\varepsilon)$ corresponds to an arc $L(\varepsilon)$ in $\{|z|<1\}$ which tends to a definite point $b(\varepsilon) \in U \cap\{|z|=1\}$, since $f(z) \rightarrow e^{i \alpha}$ on $\Delta(\varepsilon)$. By a well-known theorem of Lindelöf [4, p. 10] then the radial limit of $f(z)$ exists at $b(\varepsilon)$ and has 
the value of $e^{i a}$.

By associating $b(\varepsilon)$ with the dyadic expansion 0 . $\varepsilon_{1} \varepsilon_{2} \varepsilon_{3} \cdots$, we see that we have found a set of points $b(\varepsilon)$ in $U \cap\{|z|=1\}$, associated with the radial limit $e^{i \alpha}$, having the power of the continuum, provided that distinct sequences of $\varepsilon$ 's correspond to distinct points $b(\varepsilon)$. To show that, let $\left\{\varepsilon_{i}\right\}$ and $\left\{\varepsilon_{i}^{\prime}\right\}$ be two distinct sequences and let $p$ be the smallest integer for which $\varepsilon_{p} \neq \varepsilon_{p}^{\prime}$. Then $C_{p}\left(\varepsilon_{p}\right)$ and $C_{p}\left(\varepsilon_{p}^{\prime}\right)$ are distinct and the corresponding crosscuts $X_{p}\left(\varepsilon_{p}\right)$ and $X_{p}\left(\varepsilon_{p}^{\prime}\right)$ subtend two disjoint (recall the structure of $\mathscr{W})$ closed $\operatorname{arcs} \Lambda_{p}$ and $\Lambda_{p}^{\prime}$ on $U \cap\{|z|=1\}$. But $b(\varepsilon)$ $\in \Lambda_{p}$ and $b\left(\varepsilon^{\prime}\right) \in \Lambda_{p}^{\prime}$ and so $b(\varepsilon) \neq b\left(\varepsilon^{\prime}\right)$.

THEOREM 2. Let $b$ be a limit point of $F$ and let $U$ be a neighborhood of $b$. The set

$$
\left\{\theta \mid e^{i \theta} \in U, f\left(e^{i \theta}\right) \text { does not exist }\right\}
$$

has the power of the continuum.

Proof. Select three distinct arcs, $C(0), C(1), C(2)$, from among the arcs $C_{n}$. Suppose a curve $\Gamma$ in $\mathscr{W}$ meets, in succession, curves $\chi$ over the arcs in the sequence

$$
C\left(\varepsilon_{1}\right), C\left(\varepsilon_{2}\right), C\left(\varepsilon_{3}\right), \cdots \quad\left(\varepsilon_{i}=0,1,2 ; \quad \varepsilon_{i} \neq \varepsilon_{i+1}\right)
$$

and crosses no other $\chi$ 's. To those curves $\chi$ in $\mathscr{W}$ which $\Gamma$ meets there corresponds a sequence of crosscuts $X_{1}, X_{2}, X_{3}, \cdots$, which subtend arcs $\Lambda_{1}, \Lambda_{2}, \Lambda_{3}, \cdots$ on $\{|z|=1\}$ satisfying the condition $\Lambda_{n+1}^{-} \subset \Lambda_{n}^{\circ}$. Also we choose $\varepsilon_{1}=0$ and $X_{1}$ fixed, in $U$, so that the image of $\Gamma$ lies in $U$. The sequence $\left\{\varepsilon_{n}\right\}$ then determines a unique point $b(\varepsilon)=\bigcap \Lambda_{n}^{o} \in U$. The radius to $b(\varepsilon)$ intersects all $X_{n}$; hence $f(z)$ has no radial limit at $b(\varepsilon)$, for $C(0), C(1), C(2)$ are all distinct and $\varepsilon_{i} \neq \varepsilon_{i+1}$. Now given the start of the sequence, $\varepsilon_{1}, \varepsilon_{2}, \cdots, \varepsilon_{p}$, there are two possible choices for $\varepsilon_{p+1}$ and the two possible arcs $\Lambda_{p+1}$ are disjoint. Thus distinct sequences $\left\{\varepsilon_{n}\right\}$ yield distinct points $b(\varepsilon)$. The set of sequences $\left\{\varepsilon_{n}\right\}$ has the power of the continuum.

Theorem 3. Let $e^{i \alpha} \in E^{\prime}$. Then the set $L(\alpha)$ is countable.

Proof. Let $U$ be a neighborhood of $e^{i \alpha}$ containing none of the points $a_{n}$. Then $\mathscr{W}$ contains a countable number of schlicht components $\mathscr{U}_{1}, \mathscr{U}_{2}, \cdots$ over $U \cap\{|w|<1\}$. Each $\mathscr{U}_{n}$ maps onto $V_{n} \subset\{|z|<1\}$, where $V_{n}$ is bounded by an $\operatorname{arc} \Lambda_{n}$ of $\{|z|=1\}$ and a crosscut of $\{|z|<1\}$ The function $f(z)$ is holomorphic on $A_{n}$ and there is just one radius, ending on $A_{n}$, associated with the radial limit $e^{i \alpha}$. Since $\mathscr{W}$ contains only this countable collection of components over $U$, the result is clear. 
We remark that if $E$ is void, then the use of a two-point set $\left\{a_{1}, a_{2}\right\}$ leads to a Blaschke product satisfying Theorem 3. With a three-point set we can satisfy both Theorem 2 and Theorem 3. Theorem 1 is of course vacuous.

\section{REFERENCES}

1. P. Koebe, Abhandlungen zur Theorie der konformen Abbildung, I. Crelle 145 (1915), 177-223.

2. - Abhandlungen zur Theorie der konformen Abbildung, II. Acta Math., 40 (1915-16), 251-290.

3. - Über die Uniformisierung beliebiger analytischer Kurven, I and II, Crelle, 138 (1910), 192-253 and 139 (1911), 251-292.

4. E. Lindelöf, Sur un principe général de l'analyse et ses applications a la théorie de la représentation conforme, Acta Soc. Sci. Fenn., 46 no. 4 (1915).

5. K. Noshiro, Cluster Sets, Ergebnisse der Mathematik and ihrer Grenzgebiete, n. F. 28, Springer, Berlin 1960.

6. K. Reidemeister, Einführung in die kombinatorische Topologie, Vieweg, Braunschweig 1932.

RICE UNIVERSITY 


\title{
PACIFIC JOURNAL OF MATHEMATICS
}

\author{
EDITORS
}

RalPh S. Phillips

Stanford University

Stanford, California

M. G. Arsove

University of Washington

Seattle 5 , Washington
A. L. Whiteman

University of Southern California Los Angeles 7, California

LOWell J. PAIGE

Unıversity of California

Los Angeles 24, California

\section{ASSOCIATE EDITORS}
E. F. BECKENBACH
D. DERRY
H. L. ROYDEN
E. G. STRAUS
T. M. CHERRY
M. OHTSUKA
E. SPANIER
F. WOLF

\section{SUPPORTING INSTITUTIONS}

\author{
UNIVERSITY OF BRITISH COLUMBIA \\ CALIFORNIA INSTITUTE OF TECHNOLOGY \\ UNIVERSITY OF CALIFORNIA \\ MONTANA STATE UNIVERSITY \\ UNIVERSITY OF NEVADA \\ NEW MEXICO STATE UNIVERSITY \\ OREGON STATE UNIVERSITY \\ UNIVERSITY OF OREGON \\ OSAKA UNIVERSITY \\ UNIVERSITY OF SOUTHERN CALIFORNIA
}

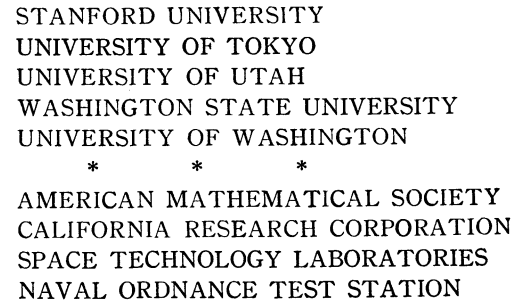

Mathematical papers intended for publication in the Pacific Journal of Mathematıcs should be typewritten (double spaced), and the author should keep a complete copy. Manuscripts may be sent to any one of the four editors. All other communications to the editors should be addressed to the managing editor, L. J. Paige at the University of California, Los Angeles 24, California.

50 reprints per author of each article are furnıshed free of charge; additional copies may be obtained at cost in multiples of 50 .

The Pacific Journal of Mathematics is published quarterly, in March, June, September, and December. Effective with Volume 13 the price per volume (4 numbers) is $\$ 18.00$; single issues, $\$ 5.00$. Special price for current issues to individual faculty members of supporting institutions and to individual members of the American Mathematical Society: $\$ 8.00$ per volume; single issues $\$ 2.50$. Back numbers are available.

Subscriptions, orders for back numbers, and changes of address should be sent to Pacific Journal of Mathematics, 103 Highland Boulevard, Berkeley 8, California.

Printed at Kokusai Bunken Insatsusha (International Academic Printing Co., Ltd.), No. 6 , 2-chome, Fujimi-cho, Chiyoda-ku, Tokyo, Japan.

PUBLISHED BY PACIFIC JOURNAL OF MATHEMATICS, A NON-PROFIT CORPORATION

The Supporting Institutions listed above contribute to the cost of publication of this Journal, but they are not owners or publishers and have no responsibility for its content or policies. 


\section{Pacific Journal of Mathematics}

\section{Vol. 12, No. $3 \quad$ March, 1962}

Alfred Aeppli, Some exact sequences in cohomology theory for Kähler

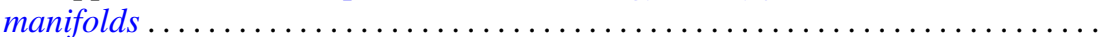

Paul Richard Beesack, On the Green's function of an N-point boundary value

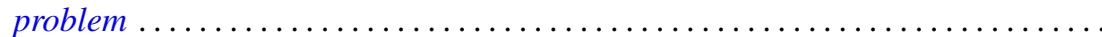

James Robert Boen, On p-automorphic p-groups....

James Robert Boen, Oscar S. Rothaus and John Griggs Thompson, Further results

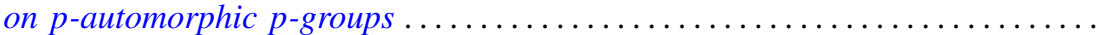

James Henry Bramble and Lawrence Edward Payne, Bounds in the Neumann problem for second order uniformly elliptic operators ..................

Chen Chung Chang and H. Jerome (Howard) Keisler, Applications of ultraproducts of pairs of cardinals to the theory of models .........................

Stephen Urban Chase, On direct sums and products of modules ................

Paul Civin, Annihilators in the second conjugate algebra of a group algebra .......

J. H. Curtiss, Polynomial interpolation in points equidistributed on the unit

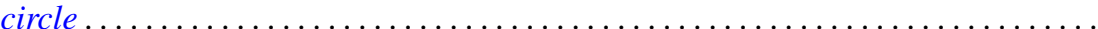

Marion K. Fort, Jr., Homogeneity of infinite products of manifolds with

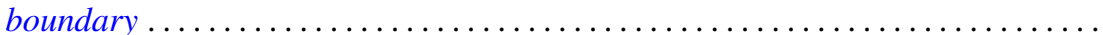

James G. Glimm, Families of induced representations . . . . . . . . . . . . . . .

Daniel E. Gorenstein, Reuben Sandler and William H. Mills, On almost-commuting

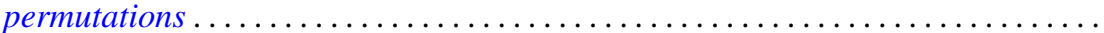

Vincent C. Harris and M. V. Subba Rao, Congruence properties of $\sigma_{r}(N) \ldots \ldots \ldots$

Harry Hochstadt, Fourier series with linearly dependent coefficients . . . . . . . . . . 925

Kenneth Myron Hoffman and John Wermer, A characterization of $C(X)$. .

Robert Weldon Hunt, The behavior of solutions of ordinary, self-adjoint differential equations of arbitrary even order...

Edward Takashi Kobayashi, A remark on the Nijenhuis tensor

David London, On the zeros of the solutions of $w^{\prime \prime}(z)+p(z) w(z)=0$

Gerald R. Mac Lane and Frank Beall Ryan, On the radial limits of Blaschke products...

T. M. MacRobert, Evaluation of an E-function when three of its upper parameters

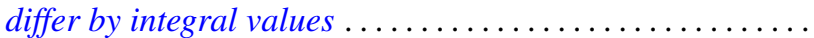

Robert W. McKelvey, The spectra of minimal self-adjoint extensions of a symmetric operator

Adegoke Olubummo, Operators of finite rank in a reflexive Banach space. .

David Alexander Pope, On the approximation of function spaces in the calculus of variations

Bernard W. Roos and Ward C. Sangren, Three spectral theorems for a pair of singular first-order differential equations...............

Arthur Argyle Sagle, Simple Malcev algebras over fields of characteristic zero .

S. C. Tang, Some theorems on the ratio of empirical distribution to the theoretical distribution

Robert Charles Thompson, Normal matrices and the normal basis in abelian number fields.

Howard Gregory Tucker, Absolute continuity of infinitely divisible distributions ... 